\title{
Enfoques mesodeliberativos: sobre la articulación institucional en las democracias deliberativas ${ }^{1}$
}

\author{
Mesodeliberative approaches: institutional structuring \\ in deliberative democracies
}

\author{
Domingo García-MarzÁ \\ Universitat Jaume I
}

Resumen. Que las democracias deliberativas sean las que ocupan hoy el centro de la reflexión democrática se debe tanto a su potencial interno como al desgaste de una realidad política alejada cada vez más de lo que pensábamos - esperábamos- de la democracia. En este contexto se enmarca el presente artículo cuya propuesta de un enfoque mesodeliberativo pretende aportar a la discusión sobre la articulación institucional de las teorías deliberativas dos aspectos íntimamente vinculados: el valor de la sociedad civil y la perspectiva de la ética en el diseño institucional. Ambos aspectos no acaban de encontrar su lugar en las diferentes iniciativas deliberativas actuales y, sin embargo, son piezas fundamentales para el logro de una teoría democrática completa y crítica.

Para defender esta propuesta daremos los siguientes pasos. En primer lugar, se justifica la necesidad de este nivel mesodeliberativo dentro del llamado enfoque sistémico de las democracias deliberativas. A continuación, ante la pregunta de qué instituciones y por qué, se introduce un concepto ampliado de sociedad civil siguiendo la propuesta de una democracia de doble vía, en discusión con la concepción habermasiana. En tercer lugar, se despliegan algunas de las aportaciones bási- cas del diseño institucional para el desarrollo de este nivel mesodeliberativo y el papel que cumple y puede cumplir en él la perspectiva ética. Por último, se esboza este papel a partir del principio kantiano de publicidad como eje central para el desarrollo de la perspectiva ética en el diseño institucional.

Palabras clave: sistemas deliberativos; democracia de doble vía; sociedad civil; diseño institucional; perspectiva ética; principio de publicidad.

Abstract. The fact that democratic reflection centres on deliberative democracies is due to both their internal potential and the erosion of a political reality, which is becoming increasingly distanced from what we either thought of as democracy or expected of it. The present article is framed in this context and puts forward a proposal for a meso-deliberative approach that aims to contribute two closely linked aspects to the discussion on the institutional articulation of deliberative theories: the value of civil society and the perspective of ethics in institutional design. Neither of these aspects has altogether found its place in today's deliberative initiatives, even though

${ }^{1}$ Este trabajo se inserta en el proyecto de Investigación FI2013-47136-C2-2-P, financiado por el Ministerio de Economía y Competitividad, así como en el Plan de Investigación P1.1B2013-24 de la Universitat Jaume I de Castellón. 
they are both essential for achieving a complete and critical democratic theory.

The paper is structured as follows: we firstly justify the need for this meso-deliberative level within what is known as the systemic approach to deliberative democracies. Then regarding the question of which institutions and why, we introduce a broad concept of civil society following the two-track democracy proposal in discussion with the Habermasian conception. We thirdly explore some of the basic contributions of institutional design to develop this meso-deliberative level, and the actual and potential role of the ethical perspective. Finally, we depict this role on the grounds of the Kantian principle of publicity as the central hub for developing an ethical perspective in institutional design.

Key words: deliberative systems; two-track democracy; civil society; institutional design; ethical perspective; principle of publicity.

\section{UN ENFOQUE SISTÉMICO PARA LA DEMOCRACIA DELIBERATIVA}

La significación e importancia de un modelo de democracia que tiene en la deliberación libre e igual, y por lo tanto en la participación y en el intercambio libre de razones, el mecanismo básico para la búsqueda de acuerdos y la toma de decisiones, no ha parado de crecer desde los años noventa ${ }^{2}$. A la justificación del papel central de la deliberación en la democracia en autores como Cohen, Habermas, Nino, Elster, etc., han seguido toda una serie de trabajos preocupados por la viabilidad práctica de estas propuestas, por las posibilidades de realización de unas ideas bien fundamentadas normativamente, pero en muchas ocasiones altamente abstractas. En esta segunda etapa los problemas no son tanto de definición como de aplicación -aunque ambos aspectos no se separen fácilmente-, de cómo acercarnos a estos ideales en sociedades complejas, funcionalmente diferenciadas, plurales y globales ${ }^{3}$.

En estas condiciones defender una participación inclusiva, libre e igual, exigida además desde nuestra autonomía moral y política, no es tarea fácil. Cuando se quieren presentar alternativas a las democracias agregativas actuales es necesario mostrar cuándo y cómo esta participación y esta deliberación

${ }^{2}$ Cfr. Para esta evolución: Bohman J., Rehg W., (eds.) Deliberative Democracy: Essays on Reason and Politics, MIT Press, Cambridge, 1997; Bohman J., "Survey Article: The Coming of Age of Deliberative Democracy", The Journal of Political Philosophy, vol.6, 4, 1998, pp. 400475; así como Martí JL., La República deliberativa, Marcial Pons, Barcelona, 2006

${ }^{3}$ Cfr. por ejemplo Benhabib S., "Deliberative Rationality and Models of Democratic Legitimacy, Constellations 1.1, 1994, pp. 26-52; Gutmann A., Thompson D., Democracy and Disagreement Harvard University Press, 1996; Dryzek J.S., Deliberative Democracy and Beyond, Oxford University Press, 2000; Parkinson J., Deliberating in the real world: Problems of legitimacy in deliberative democracy, Oxford University Press, 2006; Bohman J., Democracy across borders, Mit Press, Cambridge, 2007 
son factibles ${ }^{4}$. En las democracias deliberativas esta preocupación por analizar las diferentes posibilidades de realización ha seguido dos caminos diferentes a la hora de acercar la realidad democrática al ideal democrático ${ }^{5}$ :

\section{- Enfoques microdeliberativos}

Las propuestas se centran en las dinámicas de comunicación interpersonal, reduciendo el número de participantes y temas para así permitir una toma efectiva de decisiones tras una deliberación que respete las condiciones de una participación libre e igual. La deliberación se produce en espacios reducidos, en dinámicas de pequeña escala, de "minipúblicos", dedicados a temas concretos cuyos resultados incidan en la solución directa de problemas o sirvan de referencia para la toma de decisiones en otras esferas más amplias, por ejemplo, ya en el sistema político. Ejemplos bien conocidos son los presupuestos participativos, jurados populares, asambleas ciudadanas, foros deliberativos, comités o consejos de expertos, etc. ${ }^{6}$

- Enfoques macrodeliberativos

La deliberación ya no se produce en pequeños grupos sino en los espacios informales y abiertos de la sociedad civil, en asociaciones y organizaciones donde los ciudadanos participan por igual, no en la toma de decisiones, pero sí en la deliberación y formación de una opinión pública que, a su vez, incide en los debates públicos parlamentarios y en las decisiones políticas. Normalmente estamos ante una perspectiva crítica, un papel opositor "fuera" y "contra" el estado, derivada de la igual participación en los procesos deliberativos informales. Ejemplos tenemos desde las asociaciones cívicas y solidarias, pasando por las organizaciones de consumidores, hasta el periodismo ciudadano y su papel en la formación de una opinión pública de calidad?

${ }^{4}$ Cfr. para las posibilidades de realización de la exigencia de participación Cortina A., Ética aplicada y democracia radical, Tecnos, Madrid, 1993; así como Chambers S., "Deliberative Democracy Theory", Annual Review of political Science, 6, 2003, pp.307-326

${ }^{5} \mathrm{Cf}$. Al respecto Hendriks C.M., "When the forum meets interest politics: Strategic uses of public deliberation", Politics and Society, 34.4, 2006, pp.571-602; así como Goodin R.E., Innovating Democracy. Democratic Theory and Practice After the Deliberative Turn, Oxford University Press, 2008

${ }^{6}$ Cfr. Fishkin JS., Democracia y deliberación, Ariel, Barcelona, 1995; Fung A., Wright E.O., Abers R., (eds.) Deepening Democracy: Institutional innovations in empowered participatory governance, Verso, London, 2003; Fung A. "Minipublics: Deliberative designs and their consequences", in Rosenberg W.S. (Ed.), Can the people govern? Deliberation, participation and Democracy, Palgrave MacMillan, Bew York, pp. 159-183, 2007

${ }^{7}$ Cfr. Habermas J., Facticidad y Validez, Trotta, Madrid, 1988; Dryzek J. S., Deliberative global politics: Discourse and democracy in a divided world, Polity Press, Cambridge, 2006; Parkinson J.R., Democracy and Public Space, Oxford University Press, 2012 
Algunas de estas estrategias se han consolidado y han dado lugar a nuevas propuestas como es el caso de la democracia asociativa y la democracia monitorizada ${ }^{8}$. Sin embargo, y aunque sea posible mostrar cómo los enfoques micro tienen un impacto en los niveles macro y viceversa, la vinculación entre ambas estrategias de las democracias deliberativas necesita mayor atención ${ }^{9}$. Si el objetivo es tomar decisiones fruto de un acuerdo entre todos los afectados tras una deliberación pública, libre e igual, ninguno de los dos caminos lo acaba de conseguir. Los enfoques "micro", restringiendo temas y personas, corren el peligro de ser elitistas y quedarse, en muchas ocasiones, aislados de los problemas reales. Por su parte, los enfoques "macro" se difuminan en una opinión pública, siempre a los pies de los medios de comunicación, con escaso poder fuera de la influencia ejercida por lo que podemos llamar, siguiendo a Beck, capital de legitimación ${ }^{10}$. Utilizando la ya clásica definición de Elster, la parte deliberativa de toma de decisiones no se acaba de integrar con la parte democrática, con la inclusión de todos los participantes ${ }^{11}$. La tensión entre deliberación y participación no parece relajarse. De nuevo el dilema weberiano entre legitimidad y efectividad ${ }^{12}$.

Este viejo problema, renovado por las democracias deliberativas, está motivando una nueva serie de trabajos centrados ahora en la exploración empírica, en cómo funcionan de hecho y no en cómo deberían funcionar los enfoques micro y macrodeliberativos, en qué está pasando realmente con estas estrategias y en experimentar y predecir qué puede llegar a ocurrir. La ya casi inabarcable bibliografía actual se ocupa, entre otros temas, de comprobar la viabilidad y efectividad de las innovaciones realizadas; de explorar condiciones que favorecen o limitan la participación libre e igual; de ensayar nuevos "experimentos" deliberativos; de comprobar la verdad o falsedad de las bondades que se predican de la participación y la deliberación, etc. ${ }^{13}$

${ }^{8}$ Cfr. Para estos modelos Hirst P., Associative Democracy, Polity Press, Cambridge, 1994; Keane J., The life and death of democracy, Simon and Schuster, 2009, así como Feenstra, R.A., Democracia monitorizada en la era de la nueva galaxia mediática: la propuesta de John Keane, Icaria, Barcelona, 2012

${ }^{9}$ Goodin R.E., Dryzek J.S., "Deliberative impacts: the macro-political uptake of mini-publics", in Politics \& Society, 34.2, 2007, pp. 219-244

${ }^{10}$ Dryzek J.,S., "Legitimacy and Economy in Deliberative Democracy", in Political Theory, 29.5., 2001, pp.651-669

${ }^{11}$ Elster J., (Comp.) La democracia deliberativa, Gedisa, Barcelona, 2000; así como del mismo autor El cemento de la sociedad, Gedisa, Barcelona, 2007

${ }^{12}$ Elstub S., "Weber's Dilemma and a Dualist Model of Deliberative and Associational Democracy", in Contemporary Political theory, vol.7, 2008, pp. 169-199

${ }^{13}$ Delli Carpini M., Cook F.L., Jacobs L.R., "Public deliberation, discursive participation, and citizen engagement: A review of the empirical literature." Annu. Rev. Polit. Sci. 7, 2004, pp. 
Con su énfasis en las investigaciones de corte empírico, este tercer paso nos ayuda a entender los problemas a los que se enfrentan las propuestas deliberativas y a descubrir los escenarios en los que puedan "funcionar", inspirando y testando iniciativas concretas de reforma institucional. Pero a estas alturas sería ingenuo esperar de estas investigaciones empíricas una validación o refutación de los presupuestos normativos, ni siquiera hablar de posibles contradicciones entre los presupuestos deliberativos y la realidad. Bien sabemos que la realidad social, política y económica, no es independiente de las ideas con las que se ha construido, que la democracia depende de lo que pensemos, esto es, esperemos de ella. La aportación empírica es fundamental siempre que se integre en un esfuerzo conjunto por demostrar la viabilidad de la propuesta, junto a la justificación normativa y a la articulación institucional. Bien conocemos la pérdida de potencial crítico que resulta de la reducción de la teoría democrática a un conjunto de análisis empíricos ${ }^{14}$.

Las investigaciones empíricas aportan abundante información que debe ser tenida en cuenta en toda discusión acerca de las posibilidades de realización del libre intercambio de razones en contextos concretos y circunstancias dadas. Pero una teoría completa de la democracia exige algo más que experimentación y análisis descriptivos. Requiere, en primer lugar, una fundamentación sólida del porqué la deliberación y la exigencia consiguiente de participación y búsqueda de acuerdos respetan "la agencia moral" de los participantes, mejor que la agregación o la competencia ${ }^{15}$. En segundo lugar, tiene que presentar y justificar una articulación institucional donde "encajen" estas ideas, esto es, pensar la democracia como un entramado institucional capaz de empoderar a los individuos para que esta deliberación sea factible. Por último, y no menos importante, debe buscar las condiciones empíricas y los contextos organizativos en los que tales acuerdos institucionales logren plasmarse. Son tres niveles analítica y metodológicamente diferenciables, pero que se deben dar en toda teoría completa de la democracia ${ }^{16}$.

315-344; así como Steiner J., The foundations of deliberative democracy: Empirical research and normative implications, Cambridge University Press, 2012

${ }^{14}$ Elstub S., "The third generation of deliberative democracy." Political studies review 8.3, 2010, pp. 291-307; así como Cohen J., Fung A., "Radical democracy”, Swiss Journal of Political Science, vol.10,n.4, 2004, pp. 23-34

15 Thompson D.F., "Deliberative Democratic Theory and Empirical Science", Annual Review of Political Science, 11, 2008, pp. 497-520, p. 498

${ }^{16}$ Bohman J., "Survey Article...", op.cit., p.422; así como García-Marzá D., "La responsabilidad por la praxis: la ética discursiva como ética aplicada", en Cortina A., García-Marza D., (eds.), Razón pública y éticas aplicadas. Los caminos de la razón práctica en una sociedad pluralista, Tecnos, Madrid, 2003, pp.159-191, p.181 
Es precisamente este nivel intermedio el foco de atención para la perspectiva y las aportaciones del enfoque mesodeliberativo, centrado en los mecanismos y acuerdos institucionales capaces de incorporar la deliberación y la participación en los diferentes ámbitos. No se trata de ocupar el lugar de la representación política, pero sí de buscar nuevos espacios y mecanismos para el diálogo y el logro de acuerdos, con su diferente grado de institucionalización, sean foros, asambleas, comités, jurados, agencias internacionales, etc. ${ }^{17}$ Un enfoque que debe integrar tanto los presupuestos normativos que definen el respeto mutuo y la deliberación libre e igual en la búsqueda de acuerdos, como su, por así decirlo, potencial de realización fáctica, contando con las capacidades y motivaciones disponibles, con los recursos y limitaciones, existentes. Capacidades que solo aparecen con toda su fuerza si somos capaces de recoger dentro de este enfoque todos los recursos de la sociedad civil ${ }^{18}$.

Esta perspectiva mesodeliberativa debe incluirse en todos los esfuerzos por presentar y defender un enfoque sistémico de la democracia deliberativa, encargado de recoger los tres niveles y de relacionar entre sí los diferentes espacios donde sea posible incorporar la deliberación y la búsqueda de acuerdos ${ }^{19}$. Se trata de entender la democracia como un complejo de instituciones, gubernamentales y no gubernamentales, interconectadas entre sí y que integran la deliberación de diferentes maneras, en una división funcional del trabajo. La democracia deliberativa se refiere entonces a esta distribución de la deliberación, a esta responsabilidad compartida por abrir espacios de participación, diálogo y búsqueda de acuerdos en todas las esferas.

La deliberación puede darse en espacios reducidos o en contextos globales; con o sin representantes; en las corporaciones internacionales o en las empresas locales; en comunidades científicas; en sindicatos; partidos políticos, etc. La idea de sistema se refiere, a este entramado interconectado de instituciones que se reparten la responsabilidad por la participación y la deliberación ${ }^{20}$. En mi opinión, la inclusión de la sociedad civil con toda su complejidad y hetero-

${ }^{17}$ Chambers S., "Rhetoric and the Public Sphere: Has Deliberative Democracy Abandoned Mass Democracy", Political Theory, 37.3, 2009, pp.323-350

${ }^{18}$ Donati P., "Relational Goods and Their Subjects: The Ferment of a New Civil Society and Civil Democracy", Recerca, 2014, n 14, 19-46.

${ }^{19}$ Cfr. para este Sistema Mansbridge J., "Everyday talk in the deliberative system", Macedo S. (ed.), Deliberative Politics, Oxford University Press, 1999; Hendriks C. M. "Integrated deliberation: Reconciling civil society's dual role in deliberative democracy." Political studies, 54.3, 2006, pp. 486-508; Parkinson J.; Mansbridge J., (eds.), Deliberative systems: deliberative democracy at the large scale. Cambridge University Press, 2012

${ }^{20}$ Cornwall A.; Coelho V.S., Spaces for change?: the politics of citizen participation in new democratic arenas, Zed Books, London, 2006 
geneidad, así como su marcado tono institucionalista, constituyen un camino prometedor para continuar con el desarrollo de la democracia deliberativa. Pero, por lo general, esta inclusión de la sociedad civil no va más allá de su papel generador de opinión pública o, a lo sumo, como espacios "informales" de participación ${ }^{21}$. Si la sociedad civil solo constituye un "circuito secundario" de la política se pierde su valor básico, como veremos en el siguiente punto.

La idea de que la plasmación de la democracia deliberativa necesita un sistema o red de instituciones que vaya más allá del sistema político, que incluya todas las esferas donde existan relaciones de poder y que sea capaz de dar razón de su interdependencia, posee un gran potencial de cambio y transformación social. Pero bien sabemos que en estas esferas el reto consiste en aclarar cómo pensar espacios de deliberación cuando no se dan las condiciones para una participación libre e igual, aspecto que, al menos formalmente, queda garantizado en el estado democrático de derecho con la igualdad jurídica. Por ejemplo, en el caso de las corporaciones y sus condiciones de trabajo ${ }^{22}$. La otra solución, la más habitual incluso en las iniciativas deliberativas, es dejar a estas instituciones de la sociedad civil "fuera" de la reflexión democrática. Dar razón de estas instituciones "desde dentro", es la tarea básica de un enfoque mesodeliberativo.

Un breve recorrido por algunos textos de Habermas nos permitirá proponer una ampliación del papel que normalmente se le asigna a la sociedad civil dentro de este enfoque sistémico.

\section{DEMOCRACIA DE DOBLE VÍA: LA DISTRIBUCIÓN DE LA DELIBERACIÓN}

El actual proceso de globalización ha mostrado bien a las claras aquello que la teoría democrática venía insistiendo desde los primeros modelos participacionistas: el estado es totalmente necesario para la construcción de una voluntad común, pero totalmente insuficiente ${ }^{23}$. En mi opinión, Habermas tiene el mé-

\footnotetext{
${ }^{21}$ Cfr. Para esta limitación Smith G., Democratic Innovations, Cambridge University Press, 2009; así como Goodin, Robert E. Innovating democracy: democratic theory and practice after the deliberative turn, op.cit, p. 256

${ }^{22}$ Macdonald, K., Macdonald T., "Democracy in a pluralist global order: Corporate power and stakeholder representation." Ethics \& international affairs, 24.1, pp.19-43, 2010; así como Meadowcroft J, "Deliberative Democracy” in Durant R.F., Fiorino J., O’Leary R., (Eds.), Environmental Governance Reconsidered: Challenges, Choices, and Opportunities, MIT Press, 2004, pp. 183-217

${ }^{23}$ Cfr. al respecto Pateman C., Participation and Democratic Theory, Cambrigde Univ.Press , 1970; Macpherson C.B.,La democracia liberal y su época, Alianza, Madrid,1987; ya en el contexto de la globalización Bohman J.,Public deliberation: Pluralism, complexity, and democracy, MIT
} 
rito de haber situado la sociedad civil en el interior del sistema democrático, asignándole incluso la fuerza crítica, pero su propuesta sigue estando demasiado centrada en el estado, en la toma de decisiones legalmente vinculantes ${ }^{24}$. Tanto es así, podríamos afirmar por nuestra parte, que su visión "purista" de la sociedad civil está limitando las posibilidades de realización de la participación y la deliberación, también en los enfoques sistémicos ${ }^{25}$. Veamos por qué.

Sin poder entrar en los entresijos de la concepción habermasiana de la política deliberativa, nos interesa recordar que su metodología consiste en la reconstrucción del saber intuitivo, de las competencias y capacidades para la resolución de problemas que disponemos como participantes en los procesos democráticos. Desde esta perspectiva del participante la teoría no tiene que describir ni prescribir, debe más bien explicitar y justificar los presupuestos del sentido que los mismos ciudadanos tienen de su participación. No hay contraposición alguna entre lo ideal y lo real, pues se trata de un "contenido normativo que de entrada viene inscrito, al menos parcialmente, en la facticidad social de los propios procesos políticos observables ${ }^{26}$. Una metodología que, como a continuación propondremos, es igualmente utilizable en el resto de instituciones de la sociedad civil.

El resultado de esta reconstrucción es su idea de una democracia de doble vía, la comprensión del proceso democrático como la suma o complementación entre el Estado, como la institucionalización jurídica de la red de discursos, negociaciones y compromisos - siempre falible y revisable-y la sociedad civil, como la base social de la opinión pública. Dicho de otra forma, los mecanismos parlamentarios (concurrencia de partidos, voto, regla de mayorías, etc.) tienen que estar siempre abiertos, porosos, a la crítica de una opinión pública generada en las asociaciones y organizaciones de la sociedad civil y encargada de vigilar al resto de discursos, puesto que representa la expresión de los intereses generalizables ${ }^{27}$.

press, 2000; Dryzek J.S. Deliberative global politics: Discourse and democracy in a divided world. Cambridge: Polity, 2006

${ }^{24}$ Parkinson J.; Mansbridge J., (eds.), Deliberative systems: deliberative democracy at the large scale, op.cit.p.9; así como Hendriks C., "The ambiguous role of civil society in deliberative democracy" Paper delivered at the Australian Political Studies Association, Canberra, Australian National University, 2002.

${ }^{25}$ Keane J., "Reflexiones sobre la sociedad civil global", en Vidal Beneyto J., Hacia una sociedad civil global, Madrid, Taurus, 2003, pp. 69-119; así como Vallespin F., "Sociedad civil y crisis de la política", Isegoría,n.13, 1996, pp.39-58; Cfr.al respecto García-Marzá D., "El poder de la sociedad civil: hacia una ética de las instituciones", en Debats, 109.4, 2010, pp.30-39

${ }^{26}$ Habermas J., Facticidad y validez, op.cit., p.363; así como del mismo autor Más allá del estado nacional, Trotta, Madrid, 1997, p.143ss

${ }^{27}$ Habermas J., Facticidad y validez, op.cit. p.443 
Con esta concepción dual de la democracia se visibiliza el valor de la sociedad civil, su significación e importancia, como parte inseparable de toda concepción de la democracia. De igual forma queda también delimitado y definido el alcance de la representación, verdadero quebradero de cabeza para los enfoques micro y macro deliberativos, para la relación entre deliberación y participación en definitiva. A juicio de Habermas, nuestra libertad comunicativa, nuestra capacidad de deliberar, de transformar y de llegar a acuerdos sobre los intereses en juego, no puede delegarse, exige una participación libre e igual. De ahí que la representación tenga un mero carácter técnico, cuya legitimidad depende siempre de "una apertura sin reservas de las deliberaciones institucionalizadas al flujo de información de una opinión pública no organizada" 28 .

A la sociedad civil así entendida le corresponde introducir "la carga de la expectativa normativa", y con ello la perspectiva crítica, dentro de la teoría de la democracia. Sin embargo, a mi juicio, el precio a pagar parece ser demasiado alto: debemos sacrificar al terreno de lo "sistémico" todos aquellos ámbitos de acción que no se estructuran gracias a la acción comunicativa, la solidaridad y la búsqueda de intereses generalizables que definen la sociedad civil. Por cierto, unos ámbitos en los que, precisamente, tienen lugar la mayoría de las relaciones sociales y se genera tanto el poder como el sentido con el que se construye su posible aceptación. Toda la dimensión política y económica queda del lado sistémico, de las interacciones posibles gracias a la automatización del medio poder y del medio dinero. Así las cosas, la cuestión que surge para el desarrollo de un sistema deliberativo que pretende dar razón de la responsabilidad por la deliberación es bien sencilla: ¿No es posible ampliar este concepto de sociedad civil para que integre también a las prácticas e instituciones que no dependen "solo" de la solidaridad y la acción comunicativa? Es decir, ¿No tiene otro valor la sociedad civil que no sea como base social de la opinión pública?

La razón de esta autolimitación de las potencialidades de la sociedad civil la encontramos en la relación entre acción comunicativa y acción estratégica como mecanismos de coordinación de la acción, por un lado, y Lebenswelt y sistema como ámbitos o esferas de acción, por otro ${ }^{29}$. Mientras que la diferencia entre comunicación y estrategia remite a la orientación de la acción -a la intención en definitiva- y en este caso quien actúa sabe perfectamente si lo hace buscando el entendimiento mutuo o su propio interés, esta disyuntiva se pierde al pasar al plano social.

\footnotetext{
${ }^{28}$ Ibídem, p. 225

${ }^{29}$ Cfr. para esta crítica McCarthy T., Ideales e ilusiones: reconstrucción y deconstrucción en la teoría crítica contemporánea, Madrid, Tecnos, 1992, p.172
} 
Pero que no exista tal certidumbre no significa que desaparezca la acción comunicativa. De hecho, no existe ningún ámbito social "libre de normas", como llega a afirmar Habermas al hablar, por ejemplo, del mercado ${ }^{30}$. Todas las interacciones, prácticas e instituciones son combinaciones, mezclas, de acciones comunicativas y estratégicas, que estarán funcionalmente diferenciadas en mayor o menor medida, pero nunca "desconectadas" del mundo de la vida. En "última instancia" dependen del sentido, de los valores y normas que la Lebenswelt se encarga de proporcionar y el lenguaje cotidiano de trasmitir. Son precisamente las instituciones quienes se encargan de realizar esta integración de comunicación y estrategia para coordinar la acción y lograr así un determinado fin o bien social, por eso requieren legitimidad si quieren generar confianza, por eso no deben perder nunca el suelo de la sociedad civil ${ }^{31}$.

Si la sociedad civil solo se entiende como una red de acciones comunicativas y, por tanto, con la solidaridad como elemento único de coordinación social, Honneth nos dice con razón que Habermas se encuentra ante dos posibilidades mutuamente excluyentes. Por una parte, tenemos a la sociedad civil como esfera propia de la acción comunicativa, vaciada de poder y a la que sólo se le reconoce influencia a través de la opinión pública. Por otra, nos vemos con esferas de poder vaciadas de sustancia normativa, el estado y la economía, donde basta la racionalidad estratégico instrumental para su integración ${ }^{32}$. Pero este dualismo no es real. Ni existen asociaciones u organizaciones sin prácticas estratégicas y relaciones de poder, ni existen instituciones que no apoyen su legitimidad en el posible acuerdo de los afectados. Como muy bien se encarga de mostrar nuestro propio lenguaje al permitirnos criticar las instituciones políticas y económicas, apoyándose en gran medida en un saber moral, normalmente implícito, que nos dice aquello que tenemos derecho a esperar de estas instituciones. A este saber moral se dirige, como veremos, la perspectiva ética en el diseño institucional.

En el caso de la economía, las consecuencias de esta exclusión aparecen en Habermas cuando se enfrenta al control del proceso de globalización y, con él, a sus nefastas consecuencias para el estado y la autonomía política ${ }^{33}$. Muchos años antes, en uno de sus primeros trabajos, ya reclamaba "un proceso de comunicación formal conducido a través de la publicidad interna de las organizaciones" ${ }^{\prime 3}$. Pero esta opción será posteriormente rechazada, pues en

\footnotetext{
${ }^{30}$ Cfr. al respecto Jütten T., "Habermas and Markets”, Constellations, 20.4, 2013, pp. 587-603.

${ }^{31}$ Cfr. Al respecto Offe C., Preuss U., "Instituciones democráticas y recursos morales", Isegoria, n.2, 1990, pp.45-75

${ }^{32}$ Honneth A., Critica del poder, Machado Libros, 2009, p.434 y ss

${ }^{33}$ Habermas J., La inclusión del otro, Paidós, Barcelona, 1999, p.101

${ }^{34}$ Habermas J., Historia y crítica de la opinión pública, G.Gili, Barcelona 1994
} 
los ámbitos donde se mueven intereses que no sean generalizables parece que no haya forma de actuar si no es "desde fuera", a través del derecho o desde la influencia de la opinión pública. Una impotencia que se agrava al enfrentarse a las grandes corporaciones, a los global players de tipo económico, verdaderos protagonistas del nivel transnacional ${ }^{35}$. A mi juicio, mientras esperamos que "el derecho coercitivo del legislador democrático" pueda domesticar a estos auténticos "agentes de injusticia", bien haríamos en pensar cómo intervenir "desde dentro" en estas instituciones económicas ${ }^{36}$.

Cualquier pretensión de reunir en un mismo enfoque de la democracia deliberativa las posibilidades existentes -y por tanto responsabilidades- para la deliberación y búsqueda de acuerdos, requiere a mi juicio, ampliar esta concepción reducida de sociedad civil que sigue frenando el desarrollo de un sistema deliberativo que sea capaz de incluir todo tipo de instituciones y dar razón de su interdependencia. Tanto las instituciones económicas como el resto de instituciones sociales deben formar parte del entramado institucional de la sociedad civil. Pensar en una posible articulación institucional exige centrarnos en una concepción dual de la democracia deliberativa, en una distribución de la responsabilidad entre el estado y la sociedad civil.

Esta ampliación es posible si en vez de limitarnos a la exclusividad de la acción comunicativa, partimos de este carácter dinámico de las instituciones y hablamos de la primacía de la acción y coordinación comunicativa ${ }^{37}$. En toda práctica social, y con ella en sus pilares básicos que son las instituciones, nos encontramos con intereses particulares (prestigio, dinero, etc.), con intereses comunes (profesionales, corporativos, etc.) y con intereses generales o universales (reconocimiento, dignidad, etc.). También en esta ampliación el ámbito moral se identifica con los intereses generalizables, pero estos representan siempre las condiciones necesarias para que pueda hablarse de un libre acuerdo en la definición y satisfacción de los demás tipos de intereses o, en su caso, de la justicia del compromiso alcanzado ${ }^{38}$.

${ }^{35}$ Habermas J, La constitución de Europa, Trotta, Madrid, 2012,

${ }^{36}$ García-Marzá D., “¿Agentes de justicia? La responsabilidad social de las empresas como factor de desarrollo", en Cortina A., Pereira G., Pobreza y libertad, Tecnos, Madrid, 2009, pp. 193-211

${ }^{37}$ Cohen J.L., Arato A., Sociedad civil y teoría politica, Fondo de Cultura económica, México, 2000, p. 462; así como Arato, Andrew. "Emergencia, declive y reconstrucción del concepto de sociedad civil. Pautas para análisis futuros." Isegoría 13, 1996, pp. 5-17, p.9

${ }^{38}$ Cfr. Para esta propuesta García-Marzá D., "Sociedad civil: una concepción radical”, Recerca. Revista de Pensament y Anàlisi, 8, 2008, pp.27-46; para estas condicioneso Chambers S., "A critical theory of civil society" in Chambers S., Kymlika Will (eds.), Alternative conceptions of civil society, Princenton University Press, 2002, pp. 90-110 
En ambas esferas la idea de la deliberación y el acuerdo libre y voluntario constituyen el horizonte al que remite toda posible legitimación, el criterio normativo que define a la sociedad civil y a sus instituciones. Pero en la sociedad civil, la representación y la participación deben reconciliarse con el logro del bien social que toda institución busca, con la generación de valor compartido en el caso de la empresa. La participación y la deliberación seguro llegan más lejos allí donde tengamos mejores condiciones de reciprocidad e igualdad, pero esto no impide que en el resto de instituciones no sea el acuerdo el criterio de validez y la base última de nuestra consideración moral de su justicia o injusticia. La proximidad o lejanía de esta idea definen la base empírica en la que se apoya la credibilidad y la confianza.

Esta ampliación del concepto de sociedad civil permite que el enfoque sistémico de la democracia deliberativa se extienda a todo tipo de instituciones y que la democracia se comprenda, por decirlo con Benhabib, como "un modelo para organizar el ejercicio público y colectivo del poder en las instituciones más importantes de la sociedad" ${ }^{39}$. Una teoría crítica de la democracia quedaría mermada de entrada si no fuéramos capaces de relacionar el poder social y económico con el poder comunicativo. Esto es, si no respondiéramos de la justicia e injusticia de las instituciones de la sociedad civil como parte de la reflexión democrática ${ }^{40}$. Pero para este fin, necesitamos saber cómo integrar democracia y eficiencia.

\section{LA PERSPECTIVA INSTITUCIONALISTA: LA ÉTICA EN EL DISEÑO INSTITUCIONAL}

Cualquier alternativa a las democracias agregacionistas, centradas en el voto como mecanismo básico de participación -por no decir exclusivo-, debe avanzar en la cuestión de cómo organizar las instituciones para que consigan responder de su legitimidad desde la idea de la deliberación y el libre acuerdo entre todas las partes implicadas y afectadas. La posible articulación institucional no consiste solo, ni básicamente, en la búsqueda de arreglos institucionales como espacios ad hoc, específicamente ideados para la participación y la deliberación. En mi opinión, la prioridad consiste más bien en diseñar espacios de deliberación "dentro de" las instituciones, espacios de participación, de diálogo y de búsqueda de acuerdos inclusivos ante los problemas existentes. Espacios públicos desde los que se responda de la necesaria pretensión

\footnotetext{
${ }^{39}$ Benhabib S., Las reivindicaciones de la cultura, Katz, Buenos Aires, 2003, p. 179

${ }^{40}$ Cfr. Keane, J., "Once tesis sobre el mercado y la sociedad civil", Recerca: revista de pensament $i$ anàlisi, 8 ,2008: 11-25
} 
de justicia que subyace a la confianza que nos merecen las instituciones. Esta reflexión centra la tarea del enfoque mesodeliberativo y, con ella, la aportación de la ética al diseño institucional ${ }^{41}$.

Ninguna teoría democrática avanza a golpe de giros sino de forma gradual y progresiva, integrando en un todo coherente las diferentes perspectivas ${ }^{42}$. $\mathrm{Al}$ igual que no podemos hablar en propiedad del giro empírico de las democracias deliberativas, tampoco podemos ahora hablar del giro institucionalista. Son momentos o fases de una teoría que se desarrolla y consolida conforme responde de los tres niveles o pasos mencionados que nos llevan de la teoría a la práctica, de la justificación de sus presupuestos normativos a las posibilidades de realización. Si bien la deliberación, y con ella nuestra capacidad de dialogar, transformar nuestros intereses y lograr acuerdos, es el núcleo común compartido, las diferentes propuestas de democracias deliberativas reflejan las diversas formas en que las teorías responden de estos tres niveles.

Para avanzar en este nivel mesodeliberativo debemos acudir a las teorías del diseño institucional, situadas dentro de lo que se ha dado en llamar "nuevo institucionalismo". Mientras que las teorías del diseño institucional nos permitirán introducir en la teoría deliberativa las posibilidades de realización, las exigencias de participación, de diálogo y búsqueda de acuerdos, nos ayudarán a definir la pretensión de validez que da sentido y legitima a todo tipo de instituciones. Veamos brevemente en qué consiste esta complementación.

Por una parte, las teorías del diseño institucional aportan a los enfoques mesodeliberativos el material necesario para articular sus propuestas, puesto que se ocupan del papel que juegan las instituciones en las diferentes esferas y de la forma en que las sociedades a su vez modelan y remodelan tales instituciones ${ }^{43}$. Las preguntas acerca del significado de una institución, de sus valores y normas, del bien social que persigue, de los mecanismos de funcionamiento, de su fundación y desarrollo, de los incentivos y restricciones, etc., tienen una intención eminentemente práctica: intervenir y mejorar las instituciones actualmente existentes; incluso, aunque difícil, proponer la creación de nuevas.

${ }^{41}$ Cfr. Para el caso de la empresa, cfr. García-Marzá D., Ética empresarial: del diálogo a la confianza, Trotta, Madrid, 2004; para la administración pública Cunill Grau, N. Repensando lo público a través de la sociedad. Nuevas formas de gestión pública y representación social. CLAD, Venezuela, 1997

${ }^{42}$ Engelken-Jorge M., "Un balance de los Giros Empírico y Retórico de la Teoría Deliberativa”, Revista de Investigaciones Políticas y Sociológicas, vol.11, n.4, 2012, pp.13-30

${ }^{43}$ Guy Peters B., El nuevo institucionalismo, Gedisa,Barcelona, 2003; así como Goodin R.E.(Comp.), Teoría del diseño institucional, Gedisa, Barcelona, 2003 
Si bien esta perspectiva parte siempre de la insuficiencia de toda explicación basada exclusivamente en actores individuales autónomos que ignoran los entornos institucionales en los que se mueven, aparece un nuevo institucionalismo que se entrecruza con las democracias deliberativas en dos cuestiones básicas. En primer lugar, por la extensión de la perspectiva institucionalista más allá de las instituciones gubernamentales, esto es, por la ampliación de su campo de análisis a la sociedad civil. En segundo lugar, por la posibilidad y necesidad de deliberar y poder cambiar nuestros intereses, puesto que estos no dejan de ser, en definitiva, fruto de las mismas instituciones en las que nos hemos socializado. De ahí el carácter dinámico y reflexivo de toda institución, de ahí su comprensión como procesos de aprendizaje. Más aún, reconocen que el "buen" diseño no es sólo una cuestión pragmática sino que deben buscarse y justificarse principios para el diseño institucional "con una resonancia moral más profunda". Otra cuestión es que sean capaces de ir más allá de la perspectiva utilitarista al hacerlo.

Pero hablar de "diseño institucional" parece, en principio, una expresión demasiado pretenciosa, por no decir peligrosa. Nos imaginamos inmediatamente a unos expertos que desde su despacho deciden aquello que hay que mantener o modificar, a quién incluir o excluir, qué suerte de incentivos proporcionar, etc. Impresión que cambia si nos vamos a la raíz latina de diseñar designare- y nos referimos así a la tarea de señalar aquellos principios capaces de convertirse en buenas razones para argumentar qué tipo de acuerdos sociales, o sistema de normas, preferimos mantener y reproducir y cuáles cambiar. Si bien diseñar o rediseñar son actividades intencionales, deben entenderse siempre como aportaciones a una deliberación pública acerca de las instituciones y su papel en la vida social. Se trata, más bien, de ordenar cada uno de los recursos con los que contamos para la construcción de este esqueleto de toda democracia que son las instituciones.

Por otra parte, si a través de las teorías del diseño institucional se avanza en la articulación de las diferentes propuestas de democracia deliberativa, también esta nos ayuda a su vez a determinar el sentido y validez de las instituciones. Desde el momento en que las instituciones son el fruto de acciones humanas intencionales, por más lejanas y anquilosadas que estén, sólo la libre aceptación de todos y cada uno de los que están envueltos en su actividad, dentro de la red de valores, normas y organizaciones que las estructuran, puede apoyar su justificación y, con ella, la necesaria confianza para que funcionen, esto es, sean eficaces en el logro de sus objetivos. De ahí que las instituciones constituyan, a mi juicio, acuerdos sociales estables y válidos para el logro de fines sociales básicos, resultado de la deliberación 
y el acuerdo libre e igual entre todas las partes implicadas y afectadas por su actividad ${ }^{44}$.

Las instituciones se crean para el logro de bienes sociales básicos y para la resolución de conflictos de acción más o menos perennes. Pero su validez, aquello que las legitima, no se mide por el grado de realización alcanzado, sea la educación, la salud, la defensa, etc., -siempre dependiente del grupo que tenga el poder para calcularlo-, sino por el acuerdo entre todos los grupos de intereses que las conforman, tras una deliberación en condiciones iguales y simétricas de participación. Si debemos tener en cuenta los resultados, estos son un contenido posible del diálogo racional que conduce al acuerdo o, en su defecto, a la negociación y el compromiso.

Entendidas las instituciones como acuerdos sociales que coordinan la conducta de los individuos, el diseño institucional se refiere a la posibilidad de intervenir en estos acuerdos y, si fuera el caso, a la creación de nuevos acuerdos institucionales. Estamos, pues, ante un criterio procedimental de justicia que actúa como un horizonte de sentido, de un ideal regulativo que define el sentido de las instituciones y que, por tanto, forma parte de su realidad. El acuerdo no representa un ideal constitutivo, sino regulativo. La proximidad o lejanía de esta idea nos define la justicia o injusticia de cada institución y, con ella, el grado de confianza que nos merece.

Pero este acuerdo debe reflejar tanto la aceptación del bien logrado como las estrategias seguidas para alcanzarlo. Todo diseño institucional debe enfrentarse a lo que Offe denomina "el carácter dual de las instituciones"45. A la hora de pensar las instituciones, de emprender la tarea de configurar, reformar o mejorar, debemos atender a dos tipos de razones. Desde la dimensión funcional las instituciones se ven como instrumentos para resolver problemas básicos, para alcanzar ciertas metas y fines sociales. Aquí las razones se refieren a la eficacia en el logro del bien social y son de tipo estratégico-instrumental. Desde la dimensión normativa, las instituciones se ven como productoras de sentido, con razones que tienen que ver con la comunicación y el entendimiento, con la aceptación recíproca de los derechos y deberes establecidos. La tensión entre ambas lógicas define el dinamismo de las instituciones, su equilibrio la validez o justicia de las mismas ${ }^{46}$. Si en la búsqueda de nuevos o

${ }^{44}$ García-Marzá, D., "La dimensión ética del diseño institucional” en González Esteban, Elsa (ed.) Ética y Gobernanza: un cosmopolitismo para el siglo XXI, Comares, Granada, 2013, pp. 31-58.

${ }^{45}$ Offe C., Las nuevas democracias, Hacer, Barcelona, 2004; así como Engfer H.J., "Reglen institutionellen Handelns", en Hubig CH., (hg.), Ethik institutionellen Handelns, op.cit., pp.104-128. PORTES A., "Instituciones y desarrollo: una revisión conceptual”, Cuadernos de economía”, vol. XXV, n.45, pp.13-52, 2006

${ }^{46}$ Offe C., Las nuevas democracias, op.cit., p.257 
mejores arreglos institucionales solo tenemos en cuenta una de las dos dimensiones, la propuesta está condenada al fracaso.

De ahí que una metodología apropiada para este diseño institucional deba integrar tanto aspectos normativos como empíricos, y esto solo es posible si abandonamos la perspectiva del observador que "desde fuera" entiende a las instituciones como hechos, independientes de la voluntad y la participación de sus miembros. Adoptando una perspectiva externa, el diseño institucional no avanza, como bien muestras los análisis empíricos de la democracia deliberativa, siempre centrados en un escaso número de ejemplos. Pensar en las instituciones no es lo mismo que pensar institucionalmente, puesto que esto último implica estar implicado o afectado por la institución, esto es, implica adoptar la perspectiva del participante, pensar y hablar desde la primera persona. Solo así es posible articular su carácter dual.

Habermas utiliza esta metodología reconstructiva o reflexiva cuando parte de las competencias o capacidades para la resolución de problemas que, como participantes en los procesos democráticos, poseemos. Se trata, como él mismo nos dice, de "recoger los principios de validez universalista en el horizonte de una constelación de valores particulares", sacar a la luz un saber presupuesto que utilizamos cuando formamos parte de esas prácticas. La teoría no tiene que describir, ni prescribir, debe más bien reconstruir el saber intuitivo que los implicados y afectados por los procesos democráticos poseen y que define el sentido de su participación. Pero Habermas, no va más allá del estado democrático de derecho en la utilización de esta metodología.

Desde mi punto de vista, la metodología propia para toda articulación institucional tiene que ver con este análisis reconstructivo, pero debe extenderse a todas las esferas de la sociedad civil como parte decisiva del sistema democrático. Esta ampliación de la metodología reconstructiva es posible si la entendemos como una hermenéutica crítica ${ }^{47}$. Hermenéutica, porque debemos explicitar la realidad de cada una de estas prácticas y de sus instituciones, derivada del fin social que persiguen: la creación de valor en el caso de la empresa, la salud en el caso de un hospital, la educación en la escuela, etc. Distintas lógicas que requieren distintas formas de coordinación de la acción y, en muchas ocasiones, un reparto desigual de cargas y beneficios. No se trata de subordinar los criterios de competitividad, de competencia, de liderazgo, etc., a la deliberación y el acuerdo, sino de buscar un equilibrio entre entendimiento y estrategia que tengamos razones para justificar ${ }^{48}$. Crítica, porque

${ }^{47}$ Cortina A., "El estatuto de la ética aplicada. Hermenéutica crítica de las actividades humanas", Isegoría, 13, 1996, pp.119-134

${ }^{48}$ Conill J, Horizontes de economía ética, Tecnos, Madrid, 2014 
solo el acuerdo libre e igual permite definir la validez de la reconciliación o equilibrio logrado entre la eficacia y la justicia.

Con esta referencia al acuerdo libre y voluntario aparece la perspectiva ética, centrada en las condiciones de reciprocidad que subyacen a un acuerdo que merezca el calificativo de justo, esto es, en las condiciones que permiten una aceptación basada en el libre intercambio de razones, no en el cálculo posible de resultados. La tarea básica de una ética aplicada al diseño institucional consiste entonces en presentar principios éticos para el diseño institucional apoyados en la reconstrucción de este saber moral que, como miembros de las diferentes instituciones, poseemos. En suma, se dirige a la infraestructura moral que toda institución posee y que le proporciona el apoyo moral que subyace a toda pretensión de legitimidad ${ }^{49}$.

La perspectiva ética no inventa este saber, se propone explicitarlo de forma sistemática para que pueda ser utilizado en la comprensión y gestión de cualquiera de las prácticas e instituciones que componen el sistema deliberativo. Un saber que nos capacita para valorar y enjuiciar la posible validez moral del acuerdo alcanzado, aunque la eficiencia en el logro del bien buscado requiera hablar de asimetrías. Como miembros de una determinada institución sabemos "siempre ya" qué podemos esperar y, por lo tanto, exigir, de en cada actuación. La tarea de la perspectiva ética consiste en explicitar esta dimensión moral en una serie de principios operativos para el diseño instituciona $1^{50}$. La dificultad radica en mantener, incluso mejorar, esta operatividad sin que por ello se pierda ni un ápice de su carácter de obligación moral, de su exigibilidad. Un ejemplo de cómo es posible integrar ambas cuestiones nos lo proporciona el principio kantiano de publicidad.

\section{EL PRINCIPIO KANTIANO DE PUBLICIDAD}

La perspectiva ética del diseño institucional se ocupa de las condiciones bajo las que puede producirse y reproducirse este acuerdo moral que subyace a la creencia en la legitimidad de las instituciones, tanto del estado como de la sociedad civil. El objetivo es mostrar que estos discursos morales -utilizando terminología habermasiana- no desaparecen cuando entramos en las distintas esferas de la sociedad civil, puesto que también en este caso se requiere el acuerdo de todos los grupos implicados y afectados, esto es, están en juego intereses generalizables. Como bien muestra el hecho de que seamos capaces de distinguir y valorar negociaciones y compromisos más o menos justos o moralmente correctos ${ }^{51}$.

${ }^{49}$ Offe C., Las nuevas democracias, op.cit., p.231

${ }^{50}$ Cfr. García-Marzá D., "la dimensión ética del diseño institucional”, op.cit,p.57

${ }^{51}$ Cfr. Mansbridge J. et al., "The Place of Self-Interest and the Role of Power in Deliberative Democracy", Journal of political philosophy 18.1, 2010, pp. 64-100 
Pero no es solo el carácter asimétrico de la participación y su relación con el bien social que se persigue la única preocupación de un enfoque mesodeliberativo. De nuevo aparecen las dificultades que han dado lugar a las propuestas micro y macrodeliberativas, pues el tamaño y complejidad de muchas de las instituciones que estructuran la sociedad civil -pensemos en las empresas trasnacionales por ejemplo- no permiten pensar en una justificación apoyada, aunque sea remotamente, en la participación de las partes implicadas o afectadas por su actividad ${ }^{52}$. Si, por una parte, en contextos globales las exigencias de legitimidad aumentan conforme se percibe el gran poder de los actores institucionales y, en muchos casos, su escasa, por no decir nula, capacidad de justificación; por otra, disminuyen al mismo tiempo las posibilidades de participación y deliberación. ¿Cómo hacer frente a esta situación? ¿Debemos abandonar la idea del acuerdo y limitarnos a hablar de resultados, de funcionalidad?

Aunque la exigencia del acuerdo constituye siempre un criterio de validez, un horizonte de sentido y una base para la legitimidad de las instituciones, no debemos ignorar que su proximidad o lejanía define la moralidad de las mismas. De ahí que "fácticamente" forme parte de toda articulación institucional. Para dar cuenta de este interfaz entre lo normativo y lo empírico, de la distancia entre la idea y su realización práctica, tanto las democracias deliberativas como las teorías del diseño institucional recurren frecuentemente al principio kantiano de publicidad pues, según reconocen, nos proporciona un principio de moralidad institucional "notablemente potente", una herramienta imprescindible para la crítica moral de las instituciones políticas, sociales y económicas ${ }^{53}$.Es la capacidad de las instituciones de obtener reconocimiento, de alcanzar legitimidad, lo que está en juego, de ahí el potencial crítico del concepto de publicidad ${ }^{54}$.

Sin embargo, para algunos de estos autores el recurso a este principio implica que la democracia deliberativa ha "moderado" su visión de la participación inclusiva en favor de este énfasis en la aceptabilidad pública ${ }^{55}$. Por así decirlo,

${ }^{52}$ Cfr, al respecto O'neill O., “Agents of Justice”, en Pogge T.W., Global Justice, Blackwell, Oxford, 2000, p.188-203; así como Splichal S., "The principle of publicity, public use of reason and social control", Media, Culture and Society, 42, 2002, pp.5-26;

${ }^{53}$ Cfr. Al respecto Goodin R.E., (ed.), Teoría del diseño institucional, op.cit.,p. 62; Gutmann A..,/ Thompson D., Democracy and Disagreement, op.cit,p.95; Bohman, J. "Citizenship and norms of publicity: wide public reason in cosmopolitan societies." Political Theory, vol. 27, n.2, 1997, pp. 176-202; Chambers S., "Behind Closed Doors: Publicity, secrecy, and the Quality of Deliberation", The Journal of Political Philosophy, 12.4, 2004, pp.389-410

${ }^{54}$ Laursen J.C.,"The subversive Kant”, Political Theory, 14, 1986, pp. 584-603; Para su relación con la exigencia de transparencia, cfr. Rodriguez Zepeda J., Estado y trasparencia. Un paseo por la filosofia política, México, IFAI, 2004

${ }^{55}$ Bohman J., "Survey Article: The Coming of Age of Deliberative Democracy", op.cit.p.400 
la deliberación deja de ser un instrumento para la toma de decisiones democráticas para pasar a convertirse en un mecanismo de control democrático. De esta forma, la exigencia del acuerdo como criterio de legitimidad deja de tener relevancia y la adquiere la justificación pública de las decisiones y acciones ya tomadas o realizadas. Lo importante es que las decisiones sean "aceptables" ante quienes se ven obligados por ellas. Incluso se llega a identificar con la incorporación de este principio un "giro liberal" en las democracias deliberativas, donde la accountability reemplazaría al núcleo moral del acuerdo ${ }^{56}$.

A mi juicio, sin embargo, es posible realizar una lectura diferente del principio kantiano de publicidad que no implique una renuncia a la participación, si somos capaces de relacionar las dos formulaciones que Kant nos ofrece en la Paz Perpetua como una misma exigencia moral en dos pasos. No es momento ni ocasión para entrar en esta lectura del principio kantiano, que ya he tenido ocasión de realizar en otro lugar, pero sí es conveniente finalizar este artículo con algunas consideraciones que muestren la operatividad y actualidad de este principio moral para el diseño institucional ${ }^{57}$. En mi opinión, muchos teóricos se han limitado a su primera formulación o formulación negativa, olvidando $-\mathrm{o}$ confundiendo con- la segunda formulación o formulación positiva ${ }^{58}$.

Mientras que el primer principio define el marco de actuación, declarando injustas todas las máximas que no puedan hacerse públicas, sencillamente porque al hacerlo provocarían el rechazo del público, eliminando con ello las condiciones de su realización -en el caso de acciones o decisiones institucionales la publicidad de la máxima socavaría su propia credibilidad-, la segunda formulación, al exigir la publicidad para "no fracasar en sus propósitos" remite directamente a la aprobación o aceptación de todos los afectados. De esta forma, la publicidad no solo exige transparencia, no solo se refiere a la apertura, también se refiere al consentimiento o acuerdo y, por tanto, exige participación. Público no solo se opone a secreto, también se opone a cerrado. Una doble exclusión que, por ejemplo, explica muy bien la fuerza actual de la corrupción $^{59}$. La transparencia suele quedar en manos de quien tiene el poder de reunir la información requerida y "darle" sentido.

${ }^{56}$ Cfr. Chambers S., "Deliberative Democratic Theory", Annual review of political science, 6.1., 2003, pp.307-326, p.308 y "Measuring publicity’s effect: reconciling empirical research and normative theory." Acta Politica, 40.2, 2005, pp. 255-266

${ }^{57}$ Garcia-Marzá D., "Kant’s Principle of Publicity: The Intrinsic Relationship between the two Formulations." Kant-Studien 103.1, 2012, pp. 96-113

${ }^{58}$ Un buen ejemplo es el de Luban D., "El principio de publicidad” en Goodin R.E., Teoría del diseño institucional, cit. p. 215

${ }^{59}$ Warren M.E., (ed.), Democracy and Trust, Cambridge University Press, 1999, pp. 42-88, p.70 
La fórmula negativa del principio de publicidad establece los mínimos de justicia que exigimos de hecho, como parte del saber intuitivo que poseemos como miembros de una institución. Cuando el principio nos dice que la acción injusta no puede ser anunciada en público, nos remite ya a la necesaria aceptación de todos los demás. Aceptación que, aunque sigue siendo a priori, forma parte del principio mismo. Pero el grado en que se ha conseguido, la concordancia de los fines de todos, si se ha logrado o no un interés generalizable, es algo que los propios actores deben decidir. Es decir, nos remite al posible acuerdo de quienes han de sufrir las consecuencias de la decisión. Si van a verse implicados, es lógico, pues, que su consentimiento sea necesario para realizar cualquier acción ${ }^{60}$. Siempre al menos que busquemos legitimidad, esto es, su respaldo y cooperación, y que no queramos o podamos imponerla por la manipulación, la coacción o la violencia.

Desde su posible articulación institucional, esta exigencia moral de la publicidad entendida como la suma de transparencia y participación es la base normativa que sustenta la creación de espacios públicos en el interior de las instituciones, donde estén representados todos los grupos de intereses implicados y afectados por la actividad institucional ${ }^{61}$. Con el nombre de Comités de Ética y Responsabilidad, de Comités de Ética Pública, de Comités Públicos Corporativos, Senados Corporativos, etc., se trata en todos los casos de diseñar espacios de participación (micro), donde estén representados todos los grupos de intereses implicados y afectados por la actividad institucional. Su función es recibir y canalizar tanto la información generada por la institución como la procedente de la opinión pública (macro), así como de vigilar y controlar el cumplimiento de los compromisos públicos adquiridos, de gestionar las líneas de denuncia y sistemas de cumplimiento, etc.

Estos espacios de participación y deliberación dentro de las instituciones pueden parecernos un logro escaso dado que la participación requerida debe apelar de nuevo a la representación, aunque ahora no se trate de una representación electoral. Pero la creciente necesidad de credibilidad y, por tanto, la progresiva vulnerabilidad de las instituciones, convierten a estas innovaciones institucionales en un instrumento potente para el cambio y transformación de las mismas. Aquello que está en juego, como muy bien supo ver Kant, es la capacidad de generar confianza que toda institución necesita.

${ }^{60}$ Cfr. al respecto Langer C., Reform nach Principien, Klett-Cotta, Stuttgart, 1986

${ }^{61}$ Cfr. Chambers S., "Rhetoric and the Public Sphere: Has Deliberative Democracy Abandoned Mass Democracy", Political Theory, op.cit. p.331; para estas nuevas posibilidades de representación, cfr. Saward M., "The wider canvas canvas: representation and democracy in state and society, Alonso S., Keane J., Merkel W., (eds.) " The future of representative democracy. Cambridge: Cambridge University, 2011, pp: 74-95. 


\section{BIBLIOGRAFÍA}

Arato Andrew. "Emergencia, declive y reconstrucción del concepto de sociedad civil. Pautas para análisis futuros." Isegoría 13, 1996, pp. 5-17

Benhabib S., "Deliberative Rationality and Models of Democratic Legitimacy, Constellations 1.1, 1994, pp. 26-52

Benhabib S., Las reivindicaciones de la cultura, Katz, Buenos Aires, 2003

Bohman J., Rehg W., (eds.) Deliberative Democracy: Essays on Reason and Politics, MIT Press, Cambridge, 1997

Bohman J., "Citizenship and norms of publicity: wide public reason in cosmopolitan societies." Political Theory, vol. 27, n.2, 1997, pp. 176-202

Bohman J., "Survey Article: The Coming of Age of Deliberative Democracy", The Journal of Political Philosophy, vol.6, 4, 1998, pp. 400-475

Bohman J., Public deliberation: Pluralism, complexity, and democracy, MIT press, 2000

Bohman, J., Democracy across borders. Mit Press, Cambridge, 2007

Chambers S., "A critical theory of civil society" in Chambers S., Kymlika Will (eds.), Alternative conceptions of civil society, Princenton University Press, 2002, pp. 90-110

Chambers S., "Deliberative Democracy Theory", Annual Review of political Science, 6, 2003, pp.307-326

Chambers S., "Behind Closed Doors: Publicity, secrecy, and the Quality of Deliberation", The Journal of Political Philosophy, 12.4, 2004, pp.389-410

Chambers S., "Measuring publicity's effect: reconciling empirical research and normative theory." Acta Politica, 40.2, 2005, pp. 255-266

Chambers S., "Rhetoric and the Public Sphere: Has Deliberative Democracy Abandoned Mass Democracy", Political Theory, 37.3, 2009, pp.323-350

Cohen J.L., Arato A., Sociedad civil y teoría política, FCE, México, 2000

Cohen J., Fung A., "Radical democracy", Swiss Journal of Political Science, vol.10,n.4, 2004, pp. 23-34

Conill J, Horizontes de economía ética, Tecnos, Madrid, 2014

Cornwall A.; Coelho V.S., Spaces for change?: the politics of citizen participation in new democratic arenas, Zed Books, London, 2006

Cortina A., Ética aplicada y democracia radical, Tecnos, Madrid, 1993

Cortina A., "El estatuto de la ética aplicada. Hermenéutica crítica de las actividades humanas", Isegoria, 13, 1996, pp.119-134 
Delli Carpini M., Cook F.L., Jacobs L.R., "Public deliberation, discursive participation, and citizen engagement: A review of the empirical literature." Annu. Rev. Polit. Sci. 7, 2004, pp. 315-344

Donati P., "Relational Goods and Their Subjects: The Ferment of a New Civil Society and Civil Democracy", Recerca, 2014, n 14, 19-46

Dryzek J.S., Deliberative Democracy and Beyond, Oxford University Press, 2000

Dryzek J.S., "Legitimacy and economy in deliberative democracy", in Political theory, 29.5, 2001, pp.651-669

Dryzek J.S., Deliberative global politics: Discourse and democracy in a divided world, Polity Press, Cambridge, 2006

Elster J., (Comp.) La democracia deliberativa, Gedisa, Barcelona, 2000

Elster J., El cemento de la sociedad, Gedisa, Barcelona, 2007

Elstub S., "The third generation of deliberative democracy." Political studies review 8.3, 2010, pp. 291-307

Elstub S., "Weber's Dilemma and a Dualist Model of Deliberative and Associational Democracy", in Contemporary Political theory, vol.7, 2008, pp. 169-199

Engelken-Jorge M., "Un balance de los Giros Empírico y Retórico de la Teoría Deliberativa", Revista de Investigaciones Políticas y Sociológicas, vol.11, n.4, 2012, pp.13-30

Feenstra, R.A., Democracia monitorizada en la era de la nueva galaxia mediática: la propuesta de John Keane, Icaria, Barcelona, 2012

Fishkin JS., Democracia y deliberación, Ariel, Barcelona, 1995

Fung A., Wright E.O., Abers R., (eds.) Deepening Democracy: Institutional innovations in empowered participatory governance, Verso, London, 2003

Fung A., "Minipublics: Deliberative designs and their consequences", in Rosenberg W.S. (Ed.), Can the people govern? Deliberation, participation and Democracy, Palgrave MacMillan, Bew York, pp. 159-183, 2007

García-Marzá D., Ética empresarial: del diálogo a la confianza, Trotta, Madrid, 2004

García-Marzá D., "Sociedad civil: una concepción radical.” Recerca. Revista de Pensament y Anàlisi, 8, 2008, pp.27-46

Garcia-Marzá D., “Kant's Principle of Publicity: The Intrinsic Relationship between the two Formulations." Kant-Studien 103.1, 2012, pp. 96-113

García-Marzá, D., "La dimensión ética del diseño institucional” en González Esteban, Elsa (ed.) Ética y Gobernanza: un cosmopolitismo para el siglo XXI, Comares, Granada, 2013, pp. 31-58

Goodin R.E., (Comp.), Teoría del diseño institucional, Gedisa, Barcelona, 2003 
Goodin R.E., Innovating Democracy. Democratic Theory and Practice After the Deliberative Turn, Oxford University Press, 2008

Goodin R.E.; Dryzek J.S., "Deliberative impacts: the macro-political uptake of mini-publics", in Politics \& society, 34.2, 2007, pp. 219-244

Gutmann A., Thompson D., Democracy and Disagreement, Harvard University Press, 1996

Guy Peters B., El nuevo institucionalismo, Gedisa, Barcelona, 2003

Habermas J., Historia y crítica de la opinión pública, G.Gili, Barcelona, 1994

Habermas J., Facticidad y Validez, Trotta, Madrid, 1988

Habermas J., La inclusión del otro, Paidós, Barcelona, 1999

Habermas J., Más allá del estado nacional, Trotta, Madrid, 1997

Habermas J, La constitución de Europa, Trotta, Madrid, 2012

Hendriks C. M., "When the forum meets interest politics: Strategic uses of public deliberation", Politics and Society, 34.4, 2006, pp.571-602

Hendriks C. M. "Integrated deliberation: Reconciling civil society's dual role in deliberative democracy." Political studies, 54.3, 2006, pp. 486-508

Hirst P., Associative Democracy, Polity Press, Cambridge, 1994

Honneth A., Crítica del poder, Machado Libros, 2009

Jütten T., "Habermas and Markets", Constellations, 20.4, 2013, pp. 587-603

Keane J., "Reflexiones sobre la sociedad civil global”, en Vidal Beneyto J., Hacia una sociedad civil global, Madrid, Taurus, 2003, pp. 69-119

Keane, J. "Once tesis sobre el mercado y la sociedad civil." Recerca: revista de pensament $i$ anàlisi, 8 ,2008: 11-25

Keane J., The life and death of democracy, Simon and Schuster, 2009

Macdonald, K., Macdonald T., "Democracy in a pluralist global order: Corporate power and stakeholder representation." Ethics \& international affairs, 24. 1, pp.19-43, 2010

Macpherson C.B.,La democracia liberal y su época, Alianza, Madrid, 1987

Mansbridge J., "Everyday talk in the deliberative system”, Macedo S. (ed.), Deliberative Politics, Oxford University Press, 1999

Mansbridge J. et al., "The Place of Self-Interest and the Role of Power in Deliberative Democracy”, Journal of political philosophy 18.1, 2010, pp. 64-100

Martí JL., La República deliberativa, Marcial Pons, Barcelona, 2006

McCarthy T., Ideales e ilusiones: reconstrucción y deconstrucción en la teoría crítica contemporánea, Madrid, Tecnos, 1992 
Meadowcroft J, "Deliberative Democracy" in Durant R.F., Fiorino J., O'Leary R., (Eds.), Environmental Governance Reconsidered: Challenges, Choices, and Opportunities, MIT Press, 2004, pp. 183-21

O’neill O., “Agents of Justice”, en Pogge T.W., Global Justice, Blackwell, Oxford, 2000, p.188-203

Offe C., Preuss U., “Instituciones democráticas y recursos morales”, Isegoria, n.2, 1990, pp.45-75

Offe C., Las nuevas democracias, Hacer, Barcelona, 2004

Parkinson J. Deliberating in the real world: Problems of legitimacy in deliberative democracy, Oxford University Press, 2006

Parkinson J.; Mansbridge J., (eds.), Deliberative systems: deliberative democracy at the large scale. Cambridge University Press, 2012

Parkinson J.R., Democracy and Public Space, Oxford University Press, 2012

Pateman C., Participation and Democratic Theory, Cambrigde Univ.Press , 1970

Saward M., "The wider canvas canvas: representation and democracy in state and society, Alonso S., Keane J., Merkel W., (eds.) "The future of representative democracy. Cambridge: Cambridge University, 2011

Smith G., Democratic Innovations, Cambridge University Press, 2009

Splichal S., "The principle of publicity, public use of reason and social control", $\mathrm{Me}$ dia, Culture and Society, 42, 2002, pp.5-26

Steiner J., The foundations of deliberative democracy: Empirical research and normative implications, Cambridge University Press, 2012

Thompson D.F., "Deliberative Democratic Theory and Empirical Research", Annual Review of political science”, 11, 2008, pp. 497-520

Vallespín F., "Sociedad civil y crisis de la política”, Isegoría, n.13, 1996, pp.39-58

Warren M.E., (ed.), Democracy and Trust, Cambridge University Press, 1999 